\title{
Avaliação do vigor das sementes de duas espécies de feijão em solo contaminado com elementos-traço
}

A contaminação do solo, não só ameaça o funcionamento do ecossistema como também a saúde humana. Os elementos-traço (ETs) são inseridos no ambiente através de diversas fontes, tais como: naturais, agrícolas, industriais, efluentes domésticos, atmosféricas e entre outras. A liberação e migração de íons de ETs no solo afetam a produção de alimentos, a segurança alimentar e os recursos naturais, colocando em risco a saúde humana. Dessa forma, o objetivo deste trabalho foi avaliar a vigorosidade do Feijão Mucuna (Mucuna pruriens) e Feijão de Porco (Canavalia ensiformis) em solo com altos teores de Zn proveniente da contaminação pela queima clandestina de lixo doméstico e eletrônico. O experimento foi realizado em casa de vegetação localizada no campus da Universidade do Estado de Santa Catarina (UDESC), no município de Lages-SC. A instalação das unidades experimentais seguiu o delineamento inteiramente casualizado composto por 5 tratamentos e 4 repetições, com cultivo de 2 espécies de feijão (Mucuna pruriens e Canavalia ensiformis). Foram preparadas 40 unidades experimentais em vasos casualizado composto por 5 tratamentos e 4 repetições, com cultivo de 2 espécies de feijão (Mucuna pruriens e Canavalia ensiformis). Foram preparadas 40 unidades experimentais em vasos
com capacidade de $1 \mathrm{~L}$, utilizando $900 \mathrm{~g}$ de solo em cada vaso. As unidades experimentais utilizadas foram: T1 - solo não contaminado (SNC) (testemunha), T2 - 75\% de SNC + $25 \%$ de solo contaminado com cinza (SCC), T3 - 50\% de SNC + 50\% SCC, T4 - 25\% SNC + 75\% SCC e T5 - 100\% SCC. Durante o período de 21 dias foi realizado o controle da umidade, mantendo a mesma em $80 \%$ da capacidade de campo. Após os 21 dias foi avaliado o índice SPAD das folhas, altura da parte aérea (APA), peso fresco (PF) e peso seco (PS). Os dados foram analisá-los separadamente para tanto foi utilizado um teste não paramétrico (Kruskal Wallis). Os elementos que apresentaram os maiores valores para o SCC foram o Cu (407,4 mg.kg-1) e o Zn ( $87.984,8$ mg.kg-1). Dentre as duas espécies de feijão, a espécie que demonstrou maior resistência a altas concentrações de Zn foi o Feijão Mucuna que apresentou emergência de plântulas em metade dos vasos, porém as raízes de ambas as espécies cresceram apenas no vaso controle, comprovando que as raízes de ambas as espécies não se desenvolvem em solos com o teor de $\mathrm{Zn}$ disponível acima de $1578 \mathrm{mg} . \mathrm{kg}$-1. O valor encontrado para Zn neste solo foi 195 vezes acima do valor de investigação disposto pela resolução CONAMA 420/2009. Demonstrando que a contaminação deste solo é oriunda de fontes antrópicas e a necessidade do gerenciamento da área contaminada.

Palavras-chave: Resíduos Sólidos; Resíduos Eletrônicos; Contaminação do Solo; Elementos-Traço.

\section{Evaluation of the vigor of seeds of two bean species in contaminated soil with trace elements} \begin{abstract}
Soil contamination not only threatens the functioning of the ecosystem, but also human health. The trace elements (ETs) are inserted into the environment through several sources, such as: natural, agricultural, industrial, domestic effluents, atmospheric and among others. The release and migration of ET ions in the soil affects food production, food security and natural resources,
putting human health at risk. Thus, the objective of this work was to evaluate the vigor of Mucuna beans (Mucuna pruriens) and Pork beans (Canavalia ensiformis) in soil with high levels of $\mathrm{Zn}$ from contamination by the clandestine burning of household and electronic waste. The experiment was carried out in a greenhouse located on the campus of the State University of Santa Catarina (UDESC), in the municipality of Lages-SC. The installation of the experimental units followed a completely randomized design consisting of 5 treatments and 4 repetitions, with the cultivation of 2 species of beans (Mucuna pruriens and Canavalia ensiformis). 40 experimental units were prepared in pots with a capacity of $1 \mathrm{~L}$, using $900 \mathrm{~g}$ of soil in each pot. The experimental units used were: T1 - uncontaminated soil (SNC) (control), T2 - 75\% SNC + 25\% contaminated soil with ash (SCC), T3 - 50\% SNC + 50\% SCC, T4 - 25\% SNC + 75\% SCC and T5 - 100\% SCC. During the 21-day period, humidity control was carried out, keeping it at $80 \%$ of the field capacity. After the 21 days, the leaf SPAD index, shoot height (APA), fresh weight (PF) and dry weight (PS) were evaluated. The data were analyzed separately for this purpose a non-parametric test (Kruskal Wallis) was used. The elements that showed the highest values for SCC were Cu (407. 4 greater resistance to high concentrations of $\mathrm{Zn}$ was the Mucuna Bean, which showed seedlin emergence in half of the roots of both species do not develop in soils with the available $\mathrm{Zn}$ content above $1578 \mathrm{mg} . \mathrm{kg}-1$. The value found for $\mathrm{Zn}$ in this soil was 195 times above the investigation value provided for by CONAMA Resolution 420/2009. Demonstrating that the contamination of this soil comes from anthropic sources and the need to manage the contaminated area.
\end{abstract}

Keywords: Solid waste; Electronic waste; Ground contamination; Trace elements.

Topic: Uso de Recursos Naturais

Reviewed anonymously in the process of blind peer

Mayara Alves Lopes (D)

Universidade do Estado de Santa Catarina, Brasil

http://lattes.cnpq.br/9988606071177614

http://orcid.org/0000-0002-2576-9057

lopes.maay@gmail.com

Giovana Soares Maciel (iD)

Universidade Estadual de Mato Grosso do Sul

http://lattes.cnpq.br/3489698331243094

http://orcid.org/0000-0002-1893-5504

giovana.smaciel@hotmail.com

\section{Fernanda Cantoni (iD}

Universidade do Estado de Santa Catarina, Brasil

http://lattes.cnpq.br/3982668147965819

http://orcid.org/0000-0002-6574-4305

cantoni.f@hotmail.com

DOI: 10.6008/CBPC2179-6858.2021.006.0049
Received: 08/06/2021

Approved: 27/06/2021
David Jose Miquelluti (D)

Universidade do Estado de Santa Catarina, Brasil

http://lattes.cnpq.br/0683203409889468

http://orcid.org/0000-0002-7369-6163

dmiquell@gmail.com

Mari Lucia Campos (iD)

Universidade do Estado de Santa Catarina, Brasil

http://lattes.cnpq.br/4291057837136509

http://orcid.org/0000-0003-3250-2067

mari.lucia03@gmail.com
Referencing this:

LOPES, M. A.; MACIEL, G. S.; CANTONI, F.; MIQUELLUTI, D. J.; CAMPOS M. L.. Avaliação do vigor das sementes de duas espécies de feijão em solo contaminado com elementos-traço. Revista Ibero Americana de Ciências Ambientais, v.12, n.6, p.591-601, 2021. DOI: http://doi.org/10.6008/CBPC2179-6858.2021.006.0049 


\section{INTRODUÇÃO}

Os elementos-traço (ETs) são inseridos no ambiente através de diversas fontes, tais como: naturais, agrícolas, industriais, efluentes domésticos, atmosféricas e outras. A composição e os teores de ETs disponíveis no solo, provenientes de fontes naturais, vai depender do tipo de rocha que este solo foi originado e das condições ambientais em que este solo está inserido (SOUZA et al., 2018).

A contaminação por ETs no meio ambiente são principalmente causadas por fontes antropogênicas, como emissões de mineração e fundição, irrigação de esgoto, lodo municipal, queima de combustível fóssil e aplicação de pesticidas e fertilizante (MUTHUSARAVANAN et al., 2018). De acordo com Huang et al. (2015), a entrada de ETs em solos agrícolas vem aumentando de forma intensa durante as últimas décadas. Porem foi apenas nos últimos anos que a contaminação de solos agrícolas pelo excesso desses elementos teve uma maior atenção dos pesquisadores em relação ao risco potencial a saúde humana (Bl et al., 2018). A liberação e migração de íons de ETs no solo contaminado podem afetar a produção de alimentos, a segurança alimentar e as águas subterrâneas, colocando assim em perigo a saúde humana (CHEN et al., 2014; YANG, 2016).

O Zn é usado em muitos setores industriais, como produção de metal e galvanização, preservação de alimentos, engenharia agroalimentar e biológica, produtos farmacêuticos, eletrônicos e muitos outros processos antropogênicos. Quando não há um controle ambiental adequado destas atividades, pode ocorrer a contaminação da água, do solo e das vegetações circundantes da área por meio do escoamento superficial e/ou por aerotransporte de poeiras e fuligens (LIU et al., 2019a; 2019b).

A queima de resíduos é uma das fontes de contaminação do solo por ETs e já foi considerada um dos métodos mais perigosos para a reciclagem não controlada de resíduos eletrônicos (ISIMEKHAI et al., 2017). Entretanto, ainda é realizada de forma clandestina tornando-se uma fonte de contaminação de ETs no meio ambiente.

Para a recuperação de solos contaminados com ETs, a fitorremediação é considerada uma estratégia ambientalmente amigável e econômica, está utiliza de espécies de plantas para a remoção estabilização e/ou redução do contaminante no solo por meio de absorção nas raízes e translocação para a parte aérea (WU et al., 2018; YANG et al., 2018).

De acordo com o estudo realizado por Vasconcelo et al. (2020), a Mucuna pruriens e a Canavalia ensiformis, popularmente conhecidas como Feijão Mucuna e Feijão de Porco, são espécies que apresentaram tolerância a altas doses do contaminante imazapic, indicando um potencial fitorremediador para solos contaminados com imazapic. O estudo realizado por Madalão et al. (2017), indica que o uso do Feijão de Porco (Canavalia ensiformis) para a biorremediação de solos tratados com sulfentrazone pode reduzir o risco de impactos ambientais causados pelo contaminante. Segundo Ferraço et al. (2017), além dessas espécies serem tolerantes a diversos herbicidas, podem ainda liberar exsudatos radiculares, que atuam ativando a microbiota do solo na decomposição dos componentes orgânicos aplicados, promovendo a bioestimulação.

Diversos trabalhos de pesquisa indicam efeito positivo da utilização de plantas em solos 
contaminados com zinco, sejam espécies florestais, como evidenciado para Corymbia citriodora tolerante à adição de até $1.500 \mathrm{mg} \mathrm{kg}^{-1}$ de zinco em solo com 81\% de argila (SILVA et al., 2016), para Mogno-africano (K. senegalensis A. Juss) que tolera apenas $20 \mathrm{mg}$ de zinco $\mathrm{dm}^{-3}$ (MATHEUS et al., 2017), ou leguminosas como o Canavalia ensiformis indicado para fitorremediar arsênio, chumbo e zinco (SILVA, 2017).

Para esta pesquisa selecionou-se a leguminosa Canavalia ensiformis, visto que na literatura existem registros que apontam uma absorção superior a 50\% dos metais presentes no solo (SILVA, 2018), e a Mucuna pruriens, que tem sido considerada uma importante alternativa de uso sustentável do solo, pois é responsável pela fixação biológica de $\mathrm{N}$, cobertura do solo, fornecimento de matéria orgânica, consequentemente melhora as características químicas, físicas, e biológicas do solo (OLIVEIRA et al., 2020). Considerando o exposto, o objetivo deste estudo foi avaliar a vigorosidade das sementes de Feijão Mucuna (Mucuna pruriens) e Feijão de Porco (Canavalia ensiformis) em solo contaminado com elementos- traço proveniente da queima clandestina de lixo doméstico e eletrônico.

\section{MATERIAIS E MÉTODOS}

Foram coletados amostra de solo contaminado com cinza (SCC) com cinza proveniente da queima de resíduos e de solo não contaminado (SNC), na região do município de Lages-SC. As amostras de solos foram coletadas na profundidade de 0-5 cm. O solo predominante na área de estudo é um Cambissolo Húmico Alumínico léptico (EMBRAPA, 2013). Após a coleta, as amostras foram secas ao ar, moídas e peneiradas em peneira de $4 \mathrm{~mm}$ para experimento com plantas e $2 \mathrm{~mm}$ para análise química.

Foram encaminhadas ao Laboratório de Análise Química do Solo - LASQ do CAV-UDESC, uma amostra do solo não contaminado e uma do solo contaminado, sendo os valores e teores obtidos apresentados na Tabela 1. Os métodos utilizados pelo LASQ seguem protocolo Tedesco et al. (1995).

Tabela 1: Valor de $\mathrm{pH}$, teor de matéria orgânica (MO) e teores disponíveis de $\mathrm{P}, \mathrm{K}, \mathrm{Na}$ e Zn para solo não contaminado (SNC) e contaminado com cinza (SCC).

\begin{tabular}{lll} 
Parâmetros & SNC & SCC \\
\hline $\mathrm{pH} \mathrm{H2O}$ & 5,5 & 5,9 \\
\hline $\mathrm{MO}$ & 3,0 & 35,5 \\
\hline $\mathrm{P}\left(\mathrm{mg} \cdot \mathrm{kg}^{-1}\right)$ & 53,3 & 207,3 \\
\hline $\mathrm{K}\left(\mathrm{mg} \cdot \mathrm{kg}^{-1}\right)$ & 106,0 & 94,0 \\
\hline $\mathrm{Na}\left(\mathrm{mg} \cdot \mathrm{kg}^{-1}\right)$ & 64,2 & 41,0 \\
\hline $\mathrm{Zn}\left(\mathrm{mg}^{-1} \mathrm{~kg}^{-1}\right)$ & 3,8 & 6312,6 \\
\hline
\end{tabular}

As amostras de SCC e SNC foram novamente moídas em grau de ágata e peneiradas em peneira com abertura de 0,074 mm e submetidas a análise de $\mathrm{Al}, \mathrm{As}, \mathrm{Ca}, \mathrm{Cr}, \mathrm{Cu}, \mathrm{Fe}, \mathrm{K}, \mathrm{Mg}, \mathrm{Mn}, \mathrm{Si}, \mathrm{Sr}$ e Zn em espectrometria de fluorescência de raios X (EDX), modelo Epislon 3 da Panalytical. Nessas análises foi incluída uma amostra certificada NIST 2709A (SRM San Joaquin Soil) e a Tabela 2 apresenta os teores obtidos para Al, As, Ca, Cr, $\mathrm{Cu}, \mathrm{Fe}, \mathrm{K}, \mathrm{Mg}, \mathrm{Mn}, \mathrm{Si}$, Sr e Zn na análise e os certificados da amostra NIST 2709a (SRM San Joaquin Soil).

O fator de contaminação (FC) foi calculado conforme Sahoo et al. (2016), utilizando a seguinte equação (1):

$$
\mathrm{FC}=\mathrm{C}_{\mathrm{SCC}} / \mathrm{C}_{\mathrm{SNC}}
$$


Onde, Cscc é o teor do elemento-traço na amostra de solo contaminado com cinza e CsNc é o teor do elemento-traço nas amostras de solo das áreas naturais (testemunhas). A classificação das áreas quanto ao valor de FC (SAHOO et al., 2016) encontra-se na Tabela 3.

Tabela 2: Teores de Al, As, Ca, Cr, Cu, Fe, K, Mg, Mn, Si, Sr e Zn para NIST analisado em EDX e os teores certificados da amostra NIST

\begin{tabular}{lll}
\hline Elemento $\left(\mathrm{mg}^{\mathrm{kg}} \mathrm{k}^{-1}\right)$ & NIST FRX $\left(\mathrm{mg}^{\mathrm{kg}} \mathrm{kg}^{-1}\right)$ & NIST Certificado $\left(\mathrm{mg}^{\mathrm{k}} \mathrm{kg}^{-1}\right)$ \\
\hline $\mathrm{Al}$ & 61937,0 & 75000,0 \\
$\mathrm{As}$ & 15,1 & 17,7 \\
$\mathrm{Ca}$ & 26765,3 & 18900,0 \\
$\mathrm{Cr}$ & 85,5 & 130,0 \\
$\mathrm{Cu}$ & 39,9 & 34,6 \\
$\mathrm{Fe}$ & 46807,7 & 35000,0 \\
$\mathrm{~K}$ & 23949,8 & 20300,0 \\
$\mathrm{Mg}$ & 20350,5 & 15000,0 \\
$\mathrm{Mn}$ & 596,6 & 538,0 \\
$\mathrm{Si}$ & 297555,5 & 296600,0 \\
$\mathrm{Sr}$ & 296,0 & 231,0 \\
$\mathrm{Zn}$ & 120,5 & 106,0 \\
\hline
\end{tabular}

Tabela 3: Classificação de acordo com o fator de contaminação (FC) para solos.

\begin{tabular}{ll}
\hline Valor FC & Nível de contaminação \\
\hline FC $<1$ & Baixo \\
$1 \leq F C<3$ & Moderado \\
$3 \leq$ FC $<6$ & Considerável \\
FC $\geq 6$ & Muito alto \\
\hline
\end{tabular}

Fonte: Sahoo et al. (2016).

Também foi calculado o índice de geoacumulação (IGEO) que é utilizado para avaliar a intensidade e o comportamento do elemento-traço no ambiente. Foi utilizado para sedimentos e agora é amplamente empregado para determinar a contaminação em solos. Essa metodologia de avaliação quantitativa, vem sendo tradicionalmente utilizada por diversos autores (REIS et al., 2020; NASCIMENTO et al., 2018). Para o cálculo do IGEO utilizou-se a seguinte equação (2) proposta por Muller (1969):

$$
\text { IGEO }=\log _{2}\left(C_{S C C} / 1,5 * C_{S N C}\right)
$$

Onde, Cscc é o teor do elemento alvo na área supostamente contaminada, CSNC é teor do elemento na área testemunha (natural).

A classificação propôs por Muller (1969) para valores de IGEO encontra-se na Tabela 4.

Tabela 4: Índice de geoacumulação (IGEO) para níveis de solos contaminados.

\begin{tabular}{|c|c|c|}
\hline Classe & Valor & Qualidade do solo \\
\hline 0 & IGEO $\leq 0$ & Não contaminada \\
\hline 1 & $0<$ IGEO $<1$ & $\begin{array}{l}\text { Não contaminada/ moderadamente } \\
\text { contaminada }\end{array}$ \\
\hline 2 & $1 \leq \mathrm{IGEO}<2$ & Moderadamente contaminada \\
\hline 3 & $2 \leq \mathrm{IGEO}<3$ & $\begin{array}{l}\text { Moderadamente/ fortemente } \\
\text { contaminada }\end{array}$ \\
\hline 4 & $3 \leq \mathrm{IGEO}<4$ & Fortemente contaminada \\
\hline 5 & $4 \leq \mathrm{IGEO}<5$ & extremamente \\
\hline 6 & $5 \leq \mathrm{IGEO}$ & Extremamente contaminada \\
\hline
\end{tabular}

Fonte: Muller (1969).

\section{Experimento Feijão Mucuna (Mucuna pruriens) e Feijão de Porco (Canavalia ensiformis)}

As sementes foram cedidas pela Universidade Estadual de Mato Grosso do Sul - Unidade Universitária de Aquidauana. Para obtenção da porcentagem de germinação das espécies foi conduzido um teste de 


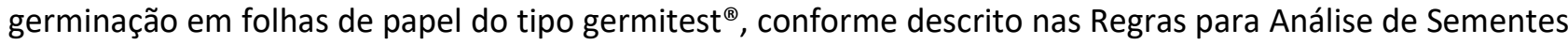
- RAS (BRASIL, 2009). A porcentagem de germinação obtida foi de $84 \%$ para Feijão de Porco e $68 \%$ para Feijão Mucuna.

O experimento foi realizado em casa de vegetação localizada no campus da Universidade do Estado de Santa Catarina (UDESC), no município de Lages-SC. A instalação das unidades experimentais seguiu o delineamento inteiramente casualizado composto por 5 tratamentos e 4 repetições, com cultivo de 2 espécies de feijão (Mucuna pruriens e Canavalia ensiformis).

Foram preparadas 40 unidades experimentais em vasos com capacidade de $1 \mathrm{~L}$, utilizando $900 \mathrm{~g}$ de solo em cada vaso. As unidades experimentais utilizadas foram: T1 - SNC (testemunha), T2 - 75\% de SNC + $25 \%$ de SCC, T3 - 50\% de SNC + 50\% SCC, T4 - 25\% SNC + 75\% SCC e T5 - 100\% SCC. As umidades das unidades experimentais foram mantidas em $80 \%$ da capacidade de campo. Em 20 vasos foram semeadas 5 sementes de Feijão Mucuna (Mucuna pruriens) e nos outros 20 vasos 5 sementes de Feijão de Porco (Canavalia ensiformis). Durante o período de 21 dias foi realizado o controle da umidade dos vasos e da emergência de plântulas.

Após os 21 dias foi avaliado o índice SPAD das folhas com uso de um clorofilômetro portátil (SPAD 502; Konica Minolta ${ }^{\circledR}$, Tóquio, Japão) e com o auxílio de um paquímetro a medida da altura da planta (APA). A seguir foram colhidas a parte aérea (PA) e raiz. As raízes foram lavadas com água destilada e deionizada. Após a PA e raiz foram secas em temperatura ambiente por um dia para obtenção do peso fresco (PF). Posteriormente, secas em estufa a $65 \stackrel{\circ}{\circ}$ até atingirem peso seco constante (PS).

Dado a diferença de comportamento entre o Feijão Mucuna (Mucuna pruriens) e o Feijão de Porco (Canavalia ensiformis), quanto ao efeito das doses, e a falta de normalidade e homogeneidade de variância, optou-se por analisá-los separadamente para tanto foi utilizado o teste não paramétrico de Kruskal Wallis. As análises estatísticas foram conduzidas utilizando-se o software R.

\section{RESULTADOS}

Teores de elementos-traço

Os resultados dos teores dos elementos nas amostras de solos encontram-se na Tabela 5.

Tabela 5: Teores de Al, As, Ca, Cr, Cu, Fe, K, Mg, Mn, Si, Sr e Zn obtidos na análise de EDX.

\begin{tabular}{lll}
\hline Elemento & SNC* & SCC \\
\hline Al (\%) & 6,2 & 4,5 \\
$\mathrm{Ca}(\%)$ & 0,3 & 0,3 \\
$\mathrm{Fe}(\%)$ & 4,0 & 5,1 \\
$\mathrm{~K}(\%)$ & 1,0 & 1,0 \\
$\mathrm{Mg}(\%)$ & 0,5 & 0,3 \\
$\mathrm{Si}(\%)$ & 33,5 & 28,0 \\
$\mathrm{As}(\mathrm{mg} / \mathrm{kg})$ & 15,1 & 7,6 \\
$\mathrm{Cr}(\mathrm{mg} / \mathrm{kg})$ & 35,9 & 41,0 \\
$\mathrm{Cu}(\mathrm{mg} / \mathrm{kg})$ & 31,9 & 407,4 \\
$\mathrm{Mn}(\mathrm{mg} / \mathrm{kg})$ & 104,6 & 255,7 \\
$\mathrm{Sr}(\mathrm{mg} / \mathrm{kg})$ & 126,8 & 156,4 \\
$\mathrm{Zn}(\mathrm{mg} / \mathrm{kg})$ & 68,3 & $87.984,8$ \\
\hline
\end{tabular}

*SNC: Solo não contaminado; SCC: Solo contaminado com cinza. 
Os fatores de contaminação (FC) para cada elemento presente no solo contaminado e o índice de geoacumulação (IGEO) de cada elemento podem ser observados na Tabela 6. De acordo com a Tabela 3, o FC de 12,8 para Cu e 1288 para Zn enquadram a área no nível muito alto de contaminação. O índice de geoacumulação do Cu se encaixa na classe 3, que de acordo com o a Tabela 4, a qualidade do solo é considerada como moderadamente e fortemente contaminada. O Zn é enquadrado na classe 6, que classifica a qualidade do solo como extremamente contaminada.

Tabela 6: Valores dos fatores de contaminação de cada elemento analisado e seu índice de geoacumulação.

\begin{tabular}{lllllllllllll}
\hline Elemento & $\mathbf{A l}$ & $\mathbf{A s}$ & $\mathbf{C a}$ & $\mathbf{C r}$ & $\mathbf{C u}$ & $\mathbf{F e}$ & $\mathbf{K}$ & $\mathbf{M g}$ & $\mathbf{M n}$ & $\mathbf{S i}$ & $\mathbf{S r}$ & $\mathbf{Z n}$ \\
\hline Valor FC* & 0,7 & 0,5 & 1,0 & 1,1 & 12,8 & 1,3 & 1,0 & 0,6 & 2,4 & 0,8 & 1,2 & 1288 \\
\hline IGEO** & 0,1 & 0,1 & 0,2 & 0,2 & 2,6 & 0,3 & 0,2 & 0,1 & 0,5 & 0,2 & 0,2 & 258,6 \\
\hline
\end{tabular}

*FC: Fator de Contaminação; **IGEO: Índice de Geoacumulação.

\section{Experimento Feijão Mucuna (Mucuna pruriens) e Feijão de Porco (Canavalia ensiformis)}

Como mostra a Figura 1 e na Tabela 7, a emergência das plântulas de Feijão Mucuna diminuiu com o aumento da porcentagem adicionada de SCC, sendo que no tratamento 50\% SCC a porcentagem de emergência das plântulas foi de apenas 25 \% das sementes implantadas. As variáveis peso fresco e peso seco de parte aérea e raiz, altura da parte aérea e comprimento de raiz sofreram uma redução considerável entre a testemunha e o tratamento $25 \%$ SCC no qual, estima-se que o teor disponível de $\mathrm{Zn}$ era de $1578 \mathrm{mg} \mathrm{kg}{ }^{-1}$.

Tabela 7: Porcentagem de emergência, peso fresco e seco de PA e raiz, altura PA e índice SPAD do Feijão de Mucuna cultivado em SCC e SNC.

\begin{tabular}{|c|c|c|c|c|c|c|c|c|}
\hline \multirow[t]{2}{*}{$\%$ de SCC } & \multirow[t]{2}{*}{ Emergência (\%) } & \multicolumn{2}{|c|}{ Peso Fresco (g) } & \multicolumn{2}{|c|}{ Peso Seco (g) } & \multirow[t]{2}{*}{ Altura PA (cm) } & \multirow[t]{2}{*}{ Comprimento Raiz $(\mathrm{cm})$} & \multirow[t]{2}{*}{ SPAD } \\
\hline & & PA & Raiz & PA & Raiz & & & \\
\hline 0 & $100 \mathrm{a}$ & $3,4 \mathrm{a}$ & $10,1 \mathrm{a}$ & $0,8 \mathrm{a}$ & $1,0 \mathrm{a}$ & $15,7 \mathrm{a}$ & $11,5 \mathrm{a}$ & $55,5 \mathrm{a}$ \\
\hline 25 & $75 b$ & $2 b$ & $0 \mathrm{~b}$ & $0,4 \mathrm{~b}$ & $0 \mathrm{~b}$ & $4,4 \mathrm{~b}$ & $\mathrm{Ob}$ & $50,8 \mathrm{~b}$ \\
\hline 50 & $25 c$ & $0,7 c$ & $0 \mathrm{~b}$ & $0,2 c$ & $\mathrm{Ob}$ & $1,7 \mathrm{c}$ & $\mathrm{Ob}$ & $47,7 \mathrm{~b}$ \\
\hline 75 & $5 d$ & $0 \mathrm{~d}$ & $\mathrm{Ob}$ & $0 \mathrm{~d}$ & $0 \mathrm{~b}$ & $0 \mathrm{~d}$ & $0 \mathrm{~b}$ & $0 \mathrm{c}$ \\
\hline 100 & $\mathrm{Od}$ & $0 \mathrm{~d}$ & $0 \mathrm{~b}$ & $0 \mathrm{~d}$ & $O b$ & $0 \mathrm{~d}$ & $O b$ & $0 \mathrm{c}$ \\
\hline
\end{tabular}

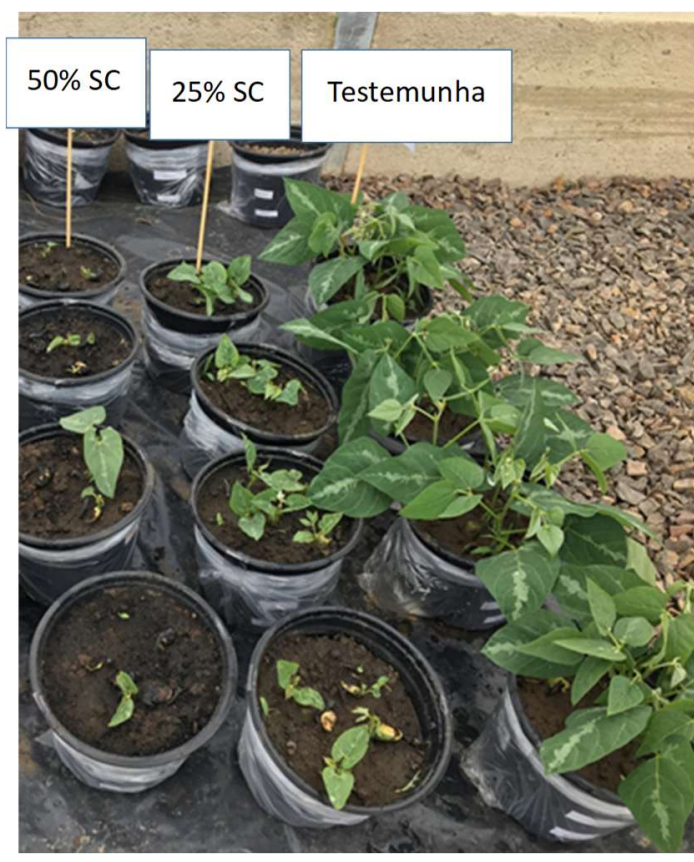

Figura 1: Emergência do Feijão Mucuna após os 21 dias, testemunha, tratamentos com $25 \%$ e $50 \%$ de solo contaminado.

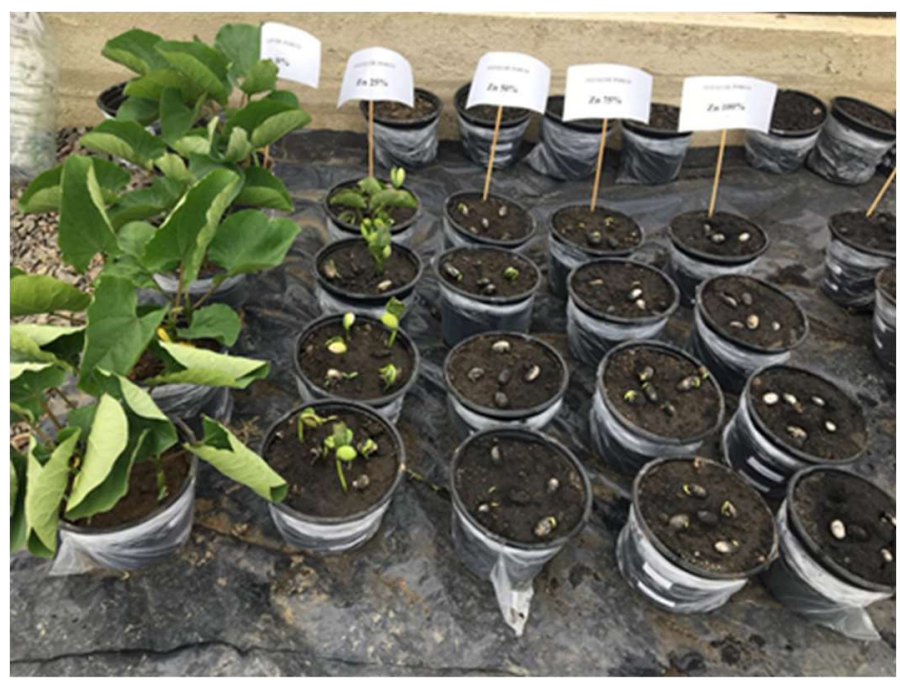

Figura 2: Emergência do Feijão de Porco após os 21 dias nos vasos controles e nos vasos com $25 \%$ de solo contaminado. 
Tabela 8: Porcentagem de emergência, peso fresco e seco de PA e raiz, altura PA e índice SPAD do Feijão de Porco cultivado em SCC e SNC.

\begin{tabular}{|c|c|c|c|c|c|c|c|c|c|}
\hline \multirow[t]{2}{*}{$\%$ de SCC } & \multirow[t]{2}{*}{$\begin{array}{l}\text { Emergência } \\
\text { (\%) }\end{array}$} & \multicolumn{2}{|c|}{ Peso Fresco (g) } & \multicolumn{2}{|c|}{ Peso Seco (g) } & \multirow[t]{2}{*}{$\begin{array}{l}\text { Altura } \\
(\mathrm{cm})\end{array}$} & \multirow[t]{2}{*}{ PA } & \multirow[t]{2}{*}{$\begin{array}{l}\text { Comprimento } \\
\text { Raiz }(\mathrm{cm})\end{array}$} & \multirow[t]{2}{*}{ SPAD } \\
\hline & & $\mathrm{PA}$ & Raiz & PA & Raiz & & & & \\
\hline 0 & $100 \mathrm{a}$ & 4,7 a & $7,3 \mathrm{a}$ & $1,6 \mathrm{a}$ & $1 a$ & $18,6 \mathrm{a}$ & & $12,6 \mathrm{a}$ & $61,2 a$ \\
\hline 25 & $60 \mathrm{~b}$ & $2,5 \mathrm{~b}$ & $0 \mathrm{~b}$ & $0,8 \mathrm{~b}$ & $0 \mathrm{~b}$ & $6,5 \mathrm{~b}$ & & $0 \mathrm{~b}$ & $50,6 \mathrm{~b}$ \\
\hline 50 & $0 \mathrm{c}$ & $0 \mathrm{c}$ & $0 \mathrm{~b}$ & $0 \mathrm{c}$ & $0 \mathrm{~b}$ & $0 \mathrm{c}$ & & $\mathrm{Ob}$ & $0 \mathrm{c}$ \\
\hline 75 & $0 \mathrm{c}$ & $0 \mathrm{c}$ & $0 \mathrm{~b}$ & $0 \mathrm{c}$ & $0 \mathrm{~b}$ & $0 \mathrm{c}$ & & $\mathrm{Ob}$ & $0 \mathrm{c}$ \\
\hline 100 & $0 \mathrm{c}$ & $0 \mathrm{c}$ & $0 \mathrm{~b}$ & $0 \mathrm{c}$ & $0 \mathrm{~b}$ & $0 \mathrm{c}$ & & $0 \mathrm{~b}$ & $0 \mathrm{c}$ \\
\hline
\end{tabular}

As sementes de Feijão de Porco germinaram apenas na testemunha e no tratamento 25\% SCC (Figura 2 e Tabela 8). Nas Figuras 3 e 4, pode-se notar que tanto para o Feijão Mucuna quanto para o Feijão de Porco, não houve a presença de raiz nos tratamentos de $25 \%$ de SCC.

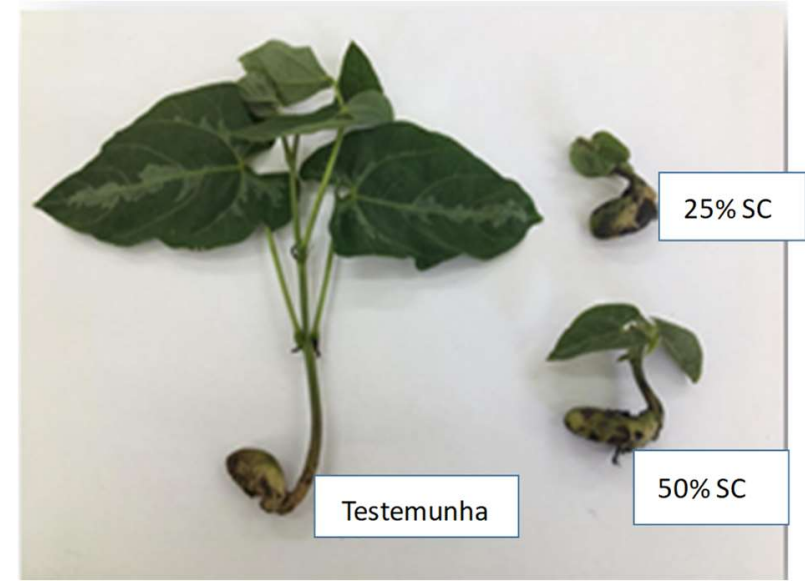

Figura 3: Parte aérea do Feijão Mucuna do vaso controle (To), do vaso com $25 \%$ de cinza (T1) e do vaso com $50 \%$ de cinza (T2).

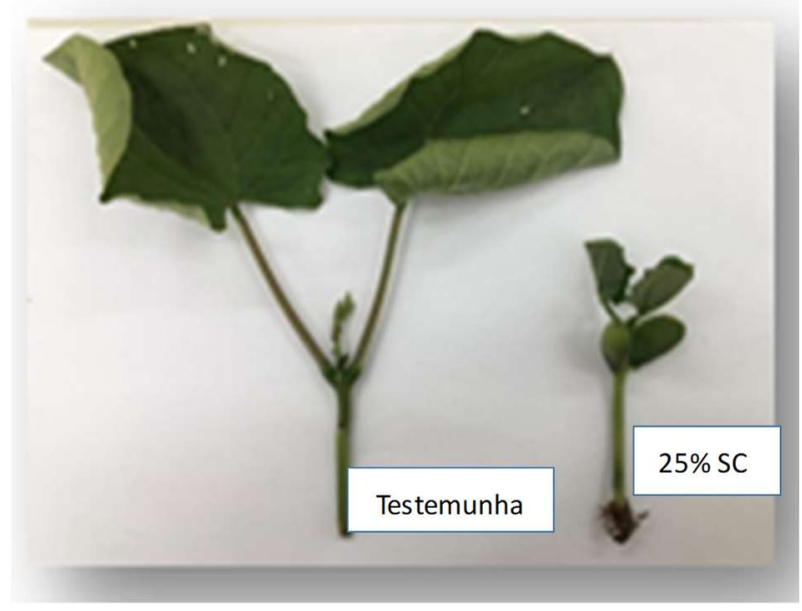

Figura 4: Parte aérea do Feijão de Porco nos tratamentos testemunhas e $25 \%$ de SCC.

\section{DISCUSSÃO}

\section{Teores de elementos-traço}

Não houve diferença estatística para os teores de $\mathrm{Al}, \mathrm{As}, \mathrm{Ca}, \mathrm{Cr}, \mathrm{Fe}, \mathrm{K}, \mathrm{Mg}, \mathrm{Mn}$, Si e Sr. Com relação ao $\mathrm{Cu}$ e Zn, os teores no SCC, foram muito superiores aos teores no SNC.

De acordo com a resolução CONAMA 420/2009, o valor de prevenção (VP) para o Cu e Zn no solo é de $60 \mathrm{mg} \cdot \mathrm{kg}^{-1}$ e $300 \mathrm{mg} \cdot \mathrm{kg}^{-1} \mathrm{e}$ o valor de investigação (VI) para áreas agrícolas é respectivamente $200 \mathrm{mg} . \mathrm{kg}^{-}$ ${ }^{1}$ e $450 \mathrm{mg} \cdot \mathrm{kg}^{-1}$. Sendo assim, os teores obtidos para Cu e Zn no solo contaminado estão acima do VI disposto pela resolução CONAMA 420/2009, classificando a qualidade do solo como classe 4 necessitando que ocorra o gerenciamento da área contaminada, pois a classe 4 é a que apresenta maior preocupação ambiental. Considerando que o teor de zinco no solo contaminado, apresentou 195 vezes acima do VI, fica evidente que houve contaminação antrópica no local de estudo.

O teor obtido para Zn na espectrometria de fluorescência de raio-x (Tabela 5) foi diferente do teor Zn apresentado no laudo da análise feita em laboratório (Tabela 1), pois a análise de EDX representa o teor total de zinco nas amostras e a análise feita no laboratório, pelo método Melinch 1, representa o teor 
disponível. Portanto, em torno de $7 \%\left(6.312,6 \mathrm{mg} \mathrm{kg}^{-1}\right)$ do $\mathrm{Zn}$ total está disponível para ser lixiviado ou absorvido pelas plantas.

O teor disponível de Zn, o enquadramento em Classe 4 pela resolução CONAMA e os valor de FC e IGEO indicam a necessidade de medidas de descontaminação e recuperação da área evitando a dispersão do poluente e a contaminação dos solos na circunvizinhas e dos recursos hídricos.

Considerando que foram encontrados restos de equipamentos eletrônicos queimados no local onde foram coletadas as amostras de cinza e comparando os resultados obtidos das análises com o estudo de Isimekhai et al. (2017) que obteve as maiores concentrações de Cu e Zn, em uma profundidade de $20-30 \mathrm{~cm}$ no solo em área onde ocorria queima de resíduos eletrônicos, pode-se concluir que o aumento dos teores de $\mathrm{Zn}$ e $\mathrm{Cu}$ no presente estudo podem ser decorrentes da queima de resíduos eletrônicos no local. Vale ressaltar que embora a concentração total de ETs no solo possa ser usada para indicar a qualidade ambiental, a gravidade da contaminação não depende da concentração total, mas também da disponibilidade e mobilidade potencial dos elementos, que nesse estudo para $\mathrm{Zn}$ também é considerada alta $(6.312,6 \mathrm{mg} \mathrm{kg}$ $\left.{ }^{1}\right)$.

\section{Experimento Feijão Mucuna (Mucuna pruriens) e Feijão de Porco (Canavalia ensiformis)}

As sementes de Feijão de Porco emergiram apenas nos vasos testemunha e no tratamento $25 \%$ SCC, isso pode ter ocorrido pois a presença de ETs em altas concentrações no meio de cultivo pode bloquear o transporte de água às sementes devido ao aumento do potencial osmótico da solução, resultando em menor absorção de água pela semente (KRANNER et al., 2011). Além disso Kalai et al. (2014), afirma que a inibição da germinação pode ocorrer devido a uma falha na mobilização do endosperma causada pelo declínio da $\alpha$ amilase, da atividade de fosfatase ácida e alcalina fosfatase.

Os únicos vasos que apresentaram raiz foram os vasos testemunha, o que pode ter sido resultante da toxicidade do Zn, que de acordo com Ansari et al. (2015) os principais efeitos morfológicos e estruturais causados pela toxicidade do $\mathrm{Zn}$ em raízes podem ser resumidos como: (i) diminuição no alongamento da raiz, biomassa e diâmetro de vaso; (ii) dano na ponta da raiz; (iii) colapso ou diminuição do cabelo radicular; (iv) aumento ou diminuição na formação de raízes laterais; (v) aumento na suberificação e lignificações e (vi) alterações na estrutura da hipoderme e endoderme.

O valor de índice SPAD diminuiu com o aumento da porcentagem de SCC, como consequência da diminuição da biossíntese de pigmentos fotossintéticos como a clorofila (a, b). A ausência de raízes e por consequência não absorção dos nutrientes ( $\mathrm{Mg}, \mathrm{N}, \mathrm{P}$ entre outros) do solo pode ter influenciado diretamente a produção de clorofila. De acordo com Azimi et al. (2020), o N é um dos nutrientes mais importantes exigidos pelas plantas e é um fator limitante no seu crescimento, pois é um componente rico em aminoácidos, proteínas, ácidos nucléicos e clorofila. A deficiência de $\mathrm{N}$ pode resultar em perturbação da proporção raizparte aérea, ramos laterais curtos, folhas pequenas, desintegração do cloroplasto e até morte.

Com relação ao crescimento das plantas, pode-se avaliar que comparado a testemunha aos outros tratamentos tiveram efeitos negativo (Fig. 3 e 4), assim como no estudo realizado por Dorneles et al. (2017), 
onde todas as concentrações acima de 2 dois $\mu \mathrm{M}$ de $\mathrm{Zn}$ causaram inibição no acúmulo de massa fresca nas plantas de feijão utilizadas. Esta redução no acúmulo de biomassa seca e fresca nas plantas de feijão, pode ser devido ao estresse oxidativo induzido pelo excesso de $\mathrm{Zn}$.

O zinco $(Z n)$ é um micronutriente essencial que afeta diversos processos metabólicos das plantas e possui uma meia-vida biológica longa (GILL, 2014). A fitotoxicidade de Zn resulta na diminuição no crescimento e desenvolvimento, metabolismo e induz a estresse oxidativo em várias espécies de plantas, como tabaco (TKALEC et al., 2014), mamona (MARQUES et al., 2014) e feijão (MENEGATTI et al., 2017). A toxicidade do zinco nas plantas limita o crescimento da raiz e da parte aérea, causa clorose nas folhas mais jovens (MALIK et al., 2011; GILL, 2014), reduz a translocação do Fe, Mn e Cu para parte aérea resultando e sintomas de deficiências desses elementos (CAMBROLLÉ et al., 2012; GILL, 2014).

\section{CONCLUSÕES}

Os elementos que apresentaram as maiores diferenças dos teores analisados para o SNC e SCC foram o $\mathrm{Cu}$ e o $\mathrm{Zn}$ sendo que o zinco apresentou um aumento mais significativo em relação ao SNC. Pode-se considerar que o elevado teor de Zn no solo ocorreu devido a queima de resíduos eletrônicos.

Nos testes de vigorosidade, a emergência do Feijão Mucuna foi maior que o Feijão de Porco. Demonstrando uma maior resistência do Feijão Mucuna aos altos teores de Zn no solo. O Feijão de Porco e Feijão Mucuna não apresentaram crescimento radicular, comprovando que para este estudo as raízes de ambas as espécies não se desenvolvem em solos com o teor de $\mathrm{Zn}$ disponível acima de $1578 \mathrm{mg} \mathrm{kg}^{-1}$.

O valor encontrado para $\mathrm{Zn}$ neste solo foi 195 vezes acima do valor de investigação disposto pela resolução CONAMA 420/2009. Demonstrando que a contaminação deste solo é oriunda de fontes antrópicas. Ficando evidente que é necessário que ocorra o isolamento e gerenciamento desta área para recuperação deste solo.

\section{REFERÊNCIAS}

ANSARI, A. A.; GILL, S. S.; GILL, R.; LANZA, G. R.; NEWMAN, L.. Phytoremediation: Management of environmental contaminants. Springer International Publishing, 2015. DOI: http://doi.org/10.1007/978-3-319-10969-5

AZIMI, S.; KAUR, T.; GANDHI, T. K.. A deep learning approach to measure stress level in plants due to Nitrogen deficiency. Measurement: Journal of the International Measurement Confederation, v.173, p.108650, 2020. DOI: http://doi.org/10.1016/i.measurement.2020.108650

BI, C.; ZHOU, Y.; CHEN, Z.; JIA, J.; BAO, X.. Heavy metals and lead isotopes in soils, road dust and leafy vegetables and health risks via vegetable consumption in the industrial areas of Shanghai, China. Science of the Total Environment, v.619, p.1349-1357, 2018. DOI:

http://doi.org/10.1016/j.scitotenv.2017.11.177

BRASIL. Ministério da Agricultura, Pecuária e Abastecimento. Secretaria de defesa agropecuária. Regras para análise de sementes. Brasília: CONAMA, 2009.
CAMBROLLÉ, J.; MANCILLA-LEYTÓN, J. M.; MUÑOZ-VALLÉS, S.; LUQUE, T.; FIGUEROA, M. E.. Zinc tolerance and accumulation in the salt-marsh shrub Halimione portulacoides. Chemosphere, v.86, n.9, p.867-874, 2012. DOI: http://doi.org/10.1016/j.chemosphere.2011.10.039

CHEN, R.; SHERBININ, A.; YE, C.; SHI, G.. China's soil pollution: Farms on the frontline. Science, v.344, n.6185, p.691, 2014. DOI:

http://doi.org/10.1126/science.344.6185.691-a

DORNELES, A. O. S.; PEREIRA, A. S.; MENEGATTI, R. D.; OLIVEIRA, L. O.; DUTRA, D. B.; DEUNER, S.. Plantulas de phaseolus vulgares I. Cultivadas sob concentrações crescentes de zinco. Revista Científica Rural, v.19, n.1, p.6069, 2017.

EMBRAPA. Centro Nacional de Pesquisa de Solos. Sistema Brasileiro de Classificação de Solos. 3 ed. Brasília: Embrapa; Rio de Janeiro: Embrapa Solos, 2013.

FERRAÇO, M.; PIRES, F. R.; BELO, A. F.; CELIN FILHO, A.; 
BONOMO, R.. Effect of population density of Canavalia ensiformis on the phytoremediation of soil contaminated with sulfentrazone. Revista Ciência Agronômica, v.48, n.1, p.32-40, 2017. DOI: http://doi.org/10.5935/1806$\underline{6690.20170004}$

GILL, M.. Heavy metal stress in plants: a review Mukti. International Journal of Advanced Research, v.2, n.6, p.1043-1055, 2014.

HUANG, Y.; LI, T.; WU, C.; HE, Z.; JAPENGA, J.; DENG, M.; YANG, $X$.. An integrated approach to assess heavy metal source apportionment in peri-urban agricultural soils. Journal of Hazardous Materials, v.299, p.540-549, 2015. DOI: http://doi.org/10.1016/j.jhazmat.2015.07.041

ISIMEKHAI, K. A.; GARELICK, H.; WATT, J.; PURCHASE, D.. Heavy metals distribution and risk assessment in soil from an informal E-waste recycling site in Lagos State, Nigeria. Environmental Science and Pollution Research, v.24, n.20, p.17206-17219, 2017. DOI: http://doi.org/10.1007/s11356017-8877-9

KALAI, T.; KHAMASSI, K.; SILVA, J. A. T.; GOUIA, H.; BEN$K A A B, L$. B.. Cadmium and copper stress affect seedling growth and enzymatic activities in germinating barley seeds. Archives of Agronomy and Soil Science, v.60, n.6, p.765783, 2014. DOI:

http://doi.org/10.1080/03650340.2013.838001

KRANNER, I.; COLVILLE, L.. Metals and seeds: Biochemical and molecular implications and their significance for seed germination. Environmental and Experimental Botany, v.72, n.1, p.93-105, 2011. DOI:

http://doi.org/10.1016/j.envexpbot.2010.05.005

LIU, J.; LI, N.; ZHANG, W.; WEI, X.; TSANG, D. C.; SUN, Y.; LUO, X.; BAO, Z.; ZHENG, W.; WANG, J.; XU, G.; HOU, L.; CHEN, Y.; FENG, Y.. Thallium contamination in farmlands and common vegetables in a pyrite-mining city and potential health risks. Environmental Pollution, v.248, p.906-915, 2019a. DOI: http://doi.org/10.1016/i.envpol.2019.02.092

LIU, J.; REN, S.; ZHOU, Y.; TSANG, D. C.; LIPPOLD, H.; WANG, J.; YIN, M.; XIAO, T.; LUO, X.; CHEN, Y.. High contamination risks of thallium and associated metal(loid)s in fluvial sediments from a steel-making area and implications for environmental management. Journal of Environmental Management, v.250, p.109513, 2019b. DOI: http://doi.org/10.1016/j.jenvman.2019.109513

MADALÃO, J. C.; SOUZA, M. F. D.; SILVA, A. A.; SILVA, D. V.; JAKELAITIS, A.; PEREIRA, G. A. M.. Action of Canavalia ensiformis in remediation of contaminated soil with sulfentrazone. Bragantia, v.76, n.2, p.292-299, 2017. DOI: http://doi.org/10.1590/1678-4499.526

MALIK, N. J.; CHAMON, A. S.; MONDOL, M. N.; ELAHI, S. F.; FAIZ, S. M. A.. Effects of different levels of zinc on growth and yield of red amaranth (Amaranthus sp.) and rice (Oryza sativa, Variety-BR49). Journal of the Bangladesh Association of Young Researchers, v.1, n.1, p.79-91, 2011.

MARQUES, M. C.; NASCIMENTO, C. W. A.. Tolerância de Mamona a zinco avaliada por fluorescência de clorofila e nutrição das plantas. Revista Brasileira de Ciência do Solo, v.38, n.3, p.850-857, 2014. DOI:

\section{http://doi.org/10.1590/S0100-06832014000300016}

MENEGATTI, R. D.; PEREIRA, A. S.; OLIVEIRA, L.; DORNELES, A. O. S.; DUTRA, D.; DEUNER, S.. Diferentes concentrações de zinco no desenvolvimento de plantas de Phaseolus vulgaris L. Evidência, v.17, n.1, p.23-32, 2017. DOI: http://doi.org/10.18593/eba.v17i1.12923

MÜLLER, G.. Index of geoaccumulation in the sediments of the Rhine River. Geojournal, v.2, n.3, p.108-118.1969.

MUTHUSARAVANAN, S.; SIVARAJASEKAR, N.; VIVEK, J. S.; PARAMASIVAN, T.; NAUSHAD, M.; PRAKASHMARAN, J.; GAYATHRI, V.; AL-DUAIJ, O. K... Phytoremediation of heavy metals: mechanisms, methods and enhancements. Environmental chemistry letters, v.16, n.4, p.1339-1359, 2018. DOI: http://doi.org/10.1007/s10311$\underline{018-0762-3}$

NASCIMENTO, L. P. D.; REIS, D. A.; ROESER, H. M. P.; SANTIAGO, A. D. F.. Avaliação geoquímica de metais em sistemas fluviais afetados por atividades antrópicas no Quadrilátero Ferrífero. Engenharia Sanitária e Ambiental, v.23, n.4, p.767-778, 2018. DOI: http://doi.org/10.1590/s1413-41522018165852

OLIVEIRA, F. K. D.; SOUZA, A. A. L.. Potencial fitorremediador do "Feijão-de-Porco" submetido a diferentes concentrações de escória siderurgia. HOLOS, v.2, p.1-13, 2020. DOI: http://doi.org/10.15628/holos.2020.6609

REIS, M. M.; SANTOS, L. D. T.; SILVA, A. J. D.; PINHO, G. P. D.; ROCHA, L. M.. Metais pesados em solos e plantas forrageiras irrigadas com água do Rio Vieira, Montes Claros, Brasil, contaminada com efluentes de esgoto. Revista Ambiente \& Água, v.15, n.2, 2020. DOI: http://doi.org/10.4136/ambiagua. 2440

SAHOO, P. K.; EQUEENUDDIN, S. K.; POWELL, M. A.. Trace elements in soils around coalmines: current scenario, impact and available techniques for management. Current Pollution Reports, v.2, n.1, p.1-14, 2016. DOI: http://doi.org/10.1007/s40726-016-0025-5

SILVA, R. F.; ANTONIOLLI, Z. I.; WEIRICH, S. W.; DELLAI, A.; MISSIO, E. L.; SCHEID, D.. Potencial da associação Pisolithus microcarpus com mudas de Corymbia citriodora em solo contaminado com zinco. Ciência Florestal, Santa Maria, v.26, n 1, p.181-191, 2016. DOI:

http://doi.org/10.5902/1980509821109

SILVA, M. R.. Potencial fitorremediador de canavalia ensiformis em neossolos flúvicos do Vale do Ribeira contaminados com chumbo, arsênio e zinco. Dissertação (Mestrado em Geociências) - Universidade Estadual de Campinas, Campinas, 2017.

SILVA, J. M. G.. Fitorremediação: um estudo da potencialidade da Canavalia ensiformis na descontaminação de solos com chumbo. Monografia (Licenciatura em Química) - Universidade Tecnológica Federal do Paraná, Londrina, 2018.

SOUZA, A. K. R.; MORASSUTI, C. Y.; DEUS, W. B.. Poluição Do Ambiente Por Metais Pesados E Utilização De Vegetais Como Bioindicadores. Acta Biomédica Brasiliensia, v.9, n.3, p.95, 2018. DOI: http://doi.org/10.18571/acbm.189 
TEDESCO, M. J.; GIANELLO, C.; BISSANI, C. A.; BOHNEN, H.; VOLKWEISS, S. J.. Análise de solo, plantas e outros materiais. 2 ed. Porto Alegre: Departamento de Solos da Universidade Federal do Rio Grande do Sul, 1995.

TKALEC, M.; ŠTEFANIĆ, P. P.; CVJETKO, P.; ŠIKIĆ, S.; PAVLICA, M.; BALEN, B.. The effects of cadmium-zinc interactions on biochemical responses in tobacco seedlings and adult plants. PLoS ONE, v.9, n.1, 2014. DOI:

http://doi.org/10.1371/journal.pone.0087582

VASCONCELO, S. M. A.; JAKELAITIS, A.; PEREIRA, L. S.; OLIVEIRA, G. S.; SOUSA, G. D.; LIMA, S. F.. Selection of tolerant species to imazapic for potential use in phytoremediation. Revista Brasileirade Ciências Agrarias, v.15, n.2, 2020. DOI:

http://doi.org/10.5039/agraria.v15i2a8075
YANG, H.. China's soil plan needs strong support. Nature, v.536, n.7617, p.375, 2016. DOI:

http://doi.org/10.1038/536375a

YANG, L. P.; ZHU, J.; WANG, P.; ZENG, J.; TAN, R.; YANG, Y. Z.; LIU, Z. M.. Effect of Cd on growth, physiological response, $\mathrm{Cd}$ subcellular distribution and chemical forms of Koelreuteria paniculata. Ecotoxicology and Environmental Safety, v.160, p.10-18, 2018. DOI:

http://doi.org/10.1016/j.ecoenv.2018.05.026

WU, M.; LUO, Q.; LIU, S.; ZHAO, Y.; LONG, Y.; PAN, Y. Screening ornamental plants to identify potential $\mathrm{Cd}$ hyperaccumulators for bioremediation. Ecotoxicology and Environmental Safety, v.162, p.35-41, 2018. DOI: http://doi.org/10.1016/j.ecoenv.2018.06.049

A CBPC - Companhia Brasileira de Produção Científica (CNPJ: 11.221.422/0001-03) detém os direitos materiais desta publicação. Os direitos referem-se à publicação do trabalho em qualquer parte do mundo, incluindo os direitos às renovações, expansões e disseminações da contribuição, bem como outros direitos subsidiários. Todos os trabalhos publicados eletronicamente poderão posteriormente ser publicados em coletâneas impressas sob coordenação da Sustenere Publishing, da Companhia Brasileira de Produção Científica e seus parceiros autorizados. Os (as) autores (as) preservam os direitos autorais, mas não têm permissão para a publicação da contribuição em outro meio, impresso ou digital, em português ou em tradução. 